\title{
Anisotropy of Positron and Electron Fluxes Measured with the Alpha Magnetic Spectrometer on the ISS
}

\author{
M. Molero ${ }^{a, *}$ and J. Casaus, C. Mañá, M.A. Velasco ${ }^{a}$ \\ ${ }^{a}$ Centro de Investigaciones Energéticas, Medioambientales y Tecnológicas (CIEMAT), \\ Avda. Complutense 40, Madrid, Spain \\ E-mail: miguel.molero@ciemat.es
}

\begin{abstract}
A measurement of the cosmic ray anisotropy on the arrival directions of elementary particles electrons and positrons has been performed in galactic coordinates by the Alpha Magnetic Spectrometer onboard the International Space Station. The analysis is based on the sample of events collected in the first 9 years of data taking. Results are consistent with isotropy and upper limits at the $95 \%$ C.I. on the dipole amplitude have been established. In particular, for energies above $16 \mathrm{GeV}$ limits of $\delta<0.36 \%$ and $\delta<1.65 \%$ are obtained for electrons and positrons respectively.
\end{abstract}

$37^{\text {th }}$ International Cosmic Ray Conference (ICRC 2021)

July 12 th - 23rd, 2021

Online - Berlin, Germany

\footnotetext{
${ }^{*}$ Presenter
} 


\section{Introduction}

As of today, AMS-02 has provided the most precise measurements of the individual positron and electron fluxes in the GeV-TeV energy range, which has revealed unexpected features that cannot be explained within the standard paradigm of the cosmic rays.

On the one hand, the positron flux [1] shows a significant excess starting in $\sim 25 \mathrm{GeV}$ followed by a sharp drop-off above $\sim 284 \mathrm{GeV}$. The spectrum is well described by the sum of a secondary component, which dominates at low energies, and a source component, which dominates at high energies, with a finite energy cutoff at $810 \mathrm{GeV}$. On the other hand, the electron flux [2] exhibits a significant excess starting from $42 \mathrm{GeV}$ compared to the lower energy trends, but the nature of this excess is different from the positron flux excess above $25 \mathrm{GeV}$. Contrary to the positrons the electrons do not present an energy cutoff below $1.9 \mathrm{TeV}$.

The origin of these features remains unclear, and a plethora of models have been proposed. In the case of positrons, the additional contribution cannot be explained by a pure secondary component and the inclusion of nearby primary sources is necessary: whether of astrophysical (pulsars) or a more exotic (dark matter) origin.

Pulsars represent the leading candidate as primary sources of positrons due to their capability to inject pairs of $e^{ \pm}$in the medium. Contributions from Geminga and Monogem are commonly used to explain the observed excess and predict anisotropies of amplitude $10^{-2}-10^{-3}$ [3-6]. Therefore, the measurement of a dipole anisotropy in the positron flux would favor the pulsar origin whereas their absence would favor the more exotic dark matter scenario.

In this context, the measurement of the arrival directions of the cosmic ray positron and electron fluxes may help to understand the observed features and, in particular, allows to explore the impact that nearby sources may imprint in the fluxes.

\section{The AMS-02 Detector}

AMS-02 is a multipurpose particle physics detector installed onboard the ISS since the 19th of May 2011. It has been designed to carry out precise measurements of charged cosmic rays in the GeV-TeV energy range. The detector has continuously collected data since its installation, with more than 180 billion of events recorded in more than 10 years. The end of the ISS is currently planned for 2028 and AMS will continue taking data until then.

The detector consists of different sub-detectors that measure the charge (Z), energy (E), momentum ( $\mathrm{p}$ ), or rigidity $(\mathrm{R}=\mathrm{p} / \mathrm{Z})$ independently. The key elements used for the present analysis are the following: a Silicon Tracker Detector (STD) with an inner tracker (L2-L8) inside a permanent magnet and two outer layers (L1 and L9), one at the top and the other at the bottom of the detector; a Transition Radiation Detector (TRD); a Time Of Flight (TOF); a Ring Imaging Cherenkov detector (RICH); and an Electromagnetic CALorimeter (ECAL). A detailed description can be found in $[7,8]$. 


\section{Electron and Positron Selection}

Selected positron and electron events are required to be relativistic downward-going particles with measured velocity $\beta \sim 1$, to have a reconstructed shower in the ECAL, with a matched track in the tracker and the TRD, charge consistent with $\mathrm{Z}=1$, and quality criteria are applied to ensure good accuracy of the track reconstruction.

Positrons and electrons are separated from protons by means of a selection based on a cut in the ECAL estimator and a template fit to the TRD response. The identification ensures a proton background below the percent level.

For the anisotropy analysis, selected events are grouped into 5 cumulative energy ranges from 16 to $500 \mathrm{GeV}$ with minimum energies $E_{\text {min }}$ : $16,25,40,65$ and $100 \mathrm{GeV}$. The final sample corresponding to the first 9 years of data taking with AMS-02 in the lowest energy range, $E_{\min }=16$ $\mathrm{GeV}$, contains $1.8 \times 10^{5}$ positrons and $2.4 \times 10^{6}$ electrons.

\section{Methodology}

The measurement of large scale anisotropies for different cosmic ray species is performed by comparing the skymap of measured events in galactic coordinates with an isotropic reference map. Both maps are created with the HEALPix scheme [9], which provides pixels of equal area in the sphere.

The reference map describes the directional response of the detector to an isotropic flux and its computation requires a detailed understanding of the detector's behavior. In particular, the precise understanding of the geographical dependences of the detector efficiencies is necessary in order to avoid possible spurious effects. More details on the construction of the isotropic reference maps can be found in [10].

In order to describe the directional dependences of the fluxes a spherical harmonic expansion is performed

$$
\Phi(\theta, \phi)=\Phi_{0}\left(1+\sum_{\ell=1} \sum_{m=-\ell}^{m=+\ell} a_{\ell m} Y_{\ell m}\right)
$$

where the $Y_{\ell m}$ are the real spherical harmonics of degree $\ell$ and order $m$, with $\ell=0,1,2$.. and $m$ $=0, \pm 1, \pm 2, \ldots, \pm \ell$, and $a_{\ell m}$ are the coefficients of the expansion, which determine the degree of the anisotropy.

The large scale anisotropy is described at first order by a dipole $(\ell=1)$ and its projection onto 3 orthogonal directions (East-West, North-South and Forward-Backward). In galactic coordinates the North-South (NS) direction is perpendicular to the galactic plane, the Forward-Backward (FB) is pointing to the galactic center, and the East-West (EW) completes the right-handed coordinate system and is contained into the galactic plane. The three dipole components can be defined as 


$$
\begin{aligned}
& \rho_{E W}=\sqrt{\frac{3}{4 \pi}} a_{1-1} \\
& \rho_{N S}=\sqrt{\frac{3}{4 \pi}} a_{10} \\
& \rho_{F B}=\sqrt{\frac{3}{4 \pi}} a_{1+1}
\end{aligned}
$$

Finally, the dipole amplitude is computed as follows

$$
\delta=\sqrt{\rho_{E W}^{2}+\rho_{N S}^{2}+\rho_{F B}^{2}}
$$

\section{Electron and Positron Anisotropy}

The measurement of the anisotropy for positron and electron events for the first 9 years of operation with AMS-02 has been found to be consistent with isotropy.

Results on the dipole components $\rho_{E W}, \rho_{N S}$ and $\rho_{F B}$ are shown in figures 1 and 3 for electrons and positrons respectively for the different energy ranges and with the 1 and 2 sigma deviations corresponding to the statistical and the statistical plus systematic uncertainties.

The dipole amplitude is computed using the three dipole components, equation 3 , as a function of the minimum energy (figures $2 \mathrm{a}$ and 4a). In particular, in the lowest energy range $E_{\min }=16 \mathrm{GeV}$ the electron and positron dipole amplitudes are $\delta_{M}^{e^{-}}=0.20 \%$ and $\delta_{M}^{e^{+}}=1.09 \%$.

Since the results are consistent with isotropy, the $95 \%$ C.I. upper limits on the dipole amplitude can be established (figures $2 \mathrm{~b}$ and $4 \mathrm{~b}$ ). In the lowest energy range $E_{\text {min }}=16 \mathrm{GeV}$, the upper limits for electrons and positrons are $\left(\delta_{U L}^{95 \%}\right)^{e^{-}}=0.36 \%$ and $\left(\delta_{U L}^{95 \%}\right)^{e^{+}}=1.65 \%$.

\section{Conclusions}

The measurement of the anisotropy in the arrival directions of cosmic ray electron and positrons in galactic coordinates has been performed with AMS-02. Results are presented for 9 years of data taking. No deviation from isotropy have been found and upper limits to the dipole amplitude are established. In particular, in the lowest energy range $E_{\text {min }}=16 \mathrm{GeV}$ the limits are $\delta<0.36 \%$ and $\delta<1.65 \%$ for electrons and positrons respectively.

AMS will continue taking data until the end of the ISS operation, currently 2028. By that time AMS measurement will be sensitive to the positron anisotropy level predicted by pulsar models. 


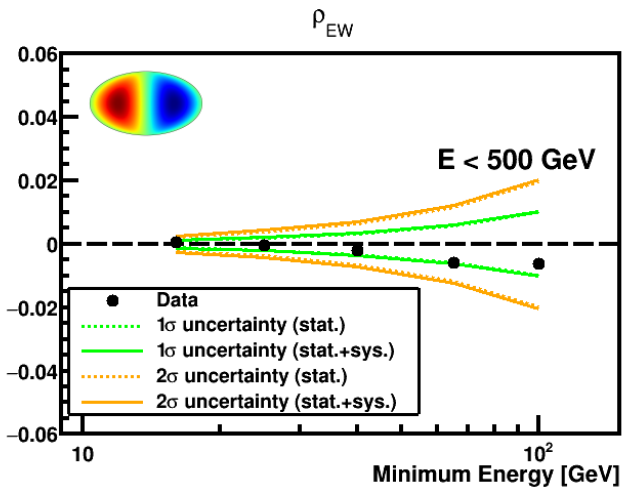

(a) East-West

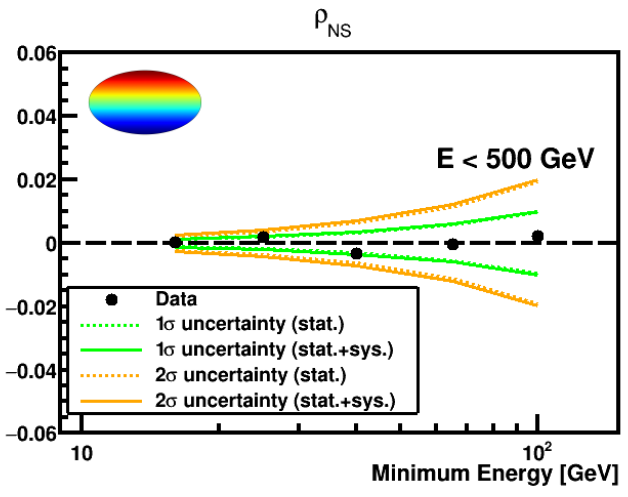

(b) North-South

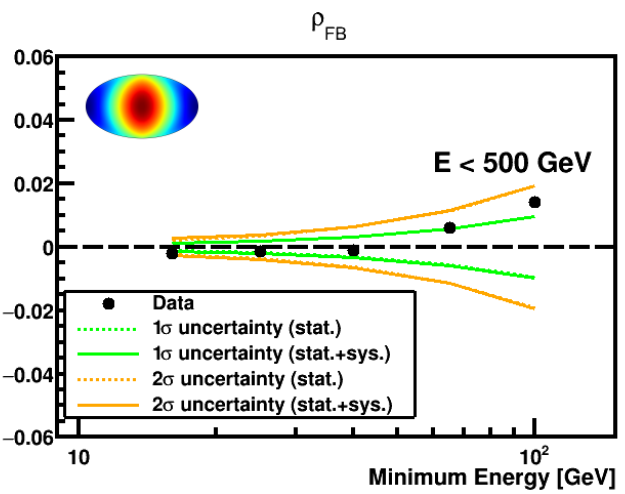

(c) Forward-Backward

Figure 1: Electron dipole components, $\rho_{E W}, \rho_{N S}$ and $\rho_{F B}$ in galactic coordinates where the 1 and 2-sigma deviations from isotropy (green and yellow, respectively) corresponding to the statistical (dotted line) and total (solid line) uncertainties are shown.

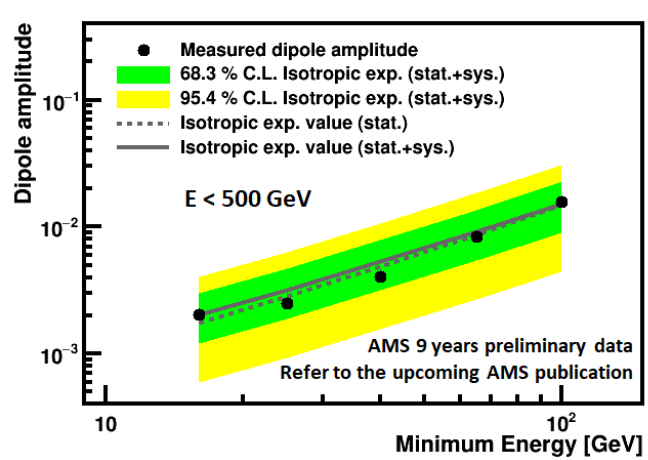

(a) Dipole Amplitude

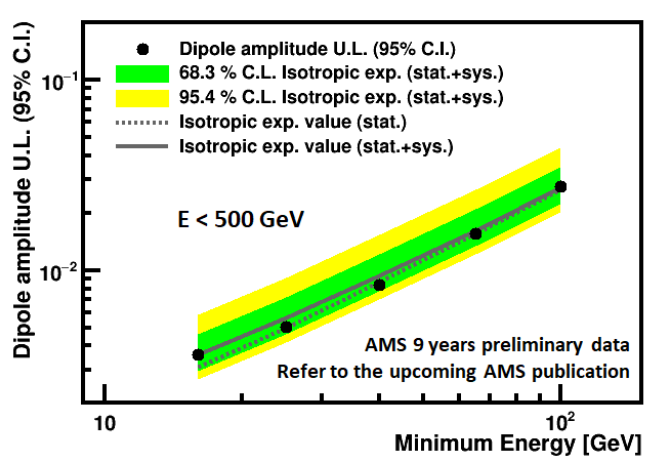

(b) Upper Limit

Figure 2: Electron measured dipole amplitude (a) and 95\% C.I. upper limit (b) as a function of the minimum energy in galactic coordinates. The 1 and 2-sigma total uncertainty bands are shown in green and yellow respectively. The expected value from isotropy considering the statistical (dotted line) and the statistical + systematic (solid line) uncertainties is also displayed. 


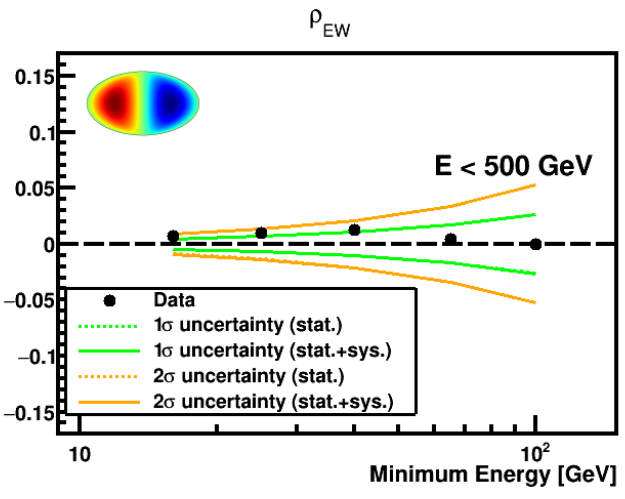

(a) East-West

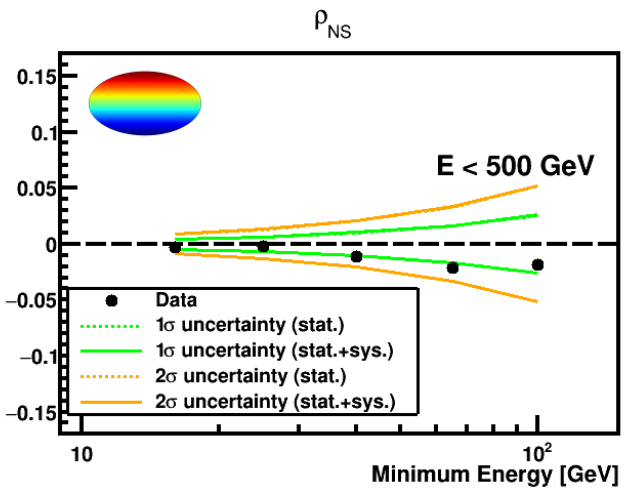

(b) North-South

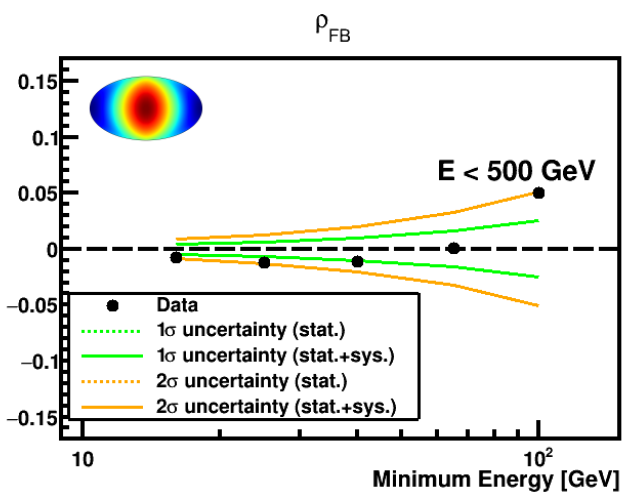

(c) Forward-Backward

Figure 3: Positron dipole components, $\rho_{E W}, \rho_{N S}$ and $\rho_{F B}$ in galactic coordinates where the 1 and 2-sigma deviations from isotropy (green and yellow, respectively) corresponding to the statistical (dotted line) and total (solid line) uncertainties are shown.

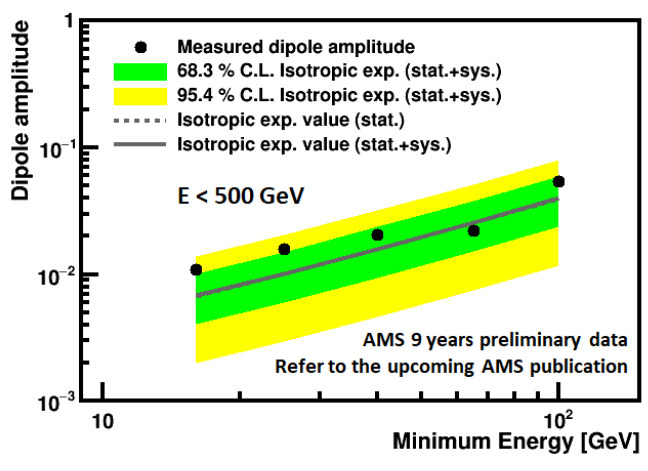

(a)

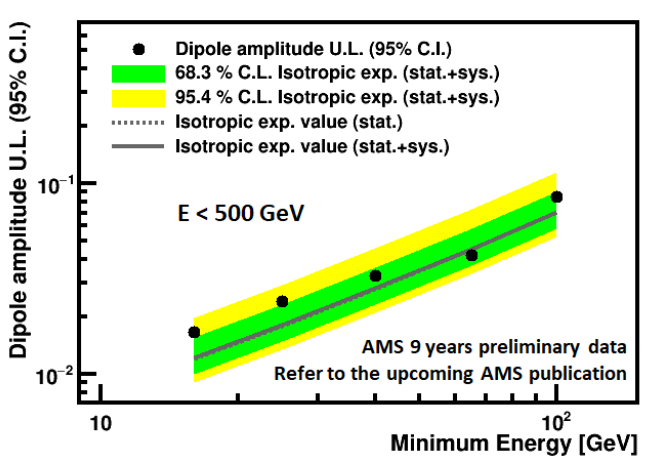

(b)

Figure 4: Positron measured dipole amplitude (a) and 95\% C.I. upper limit (b) as a function of the minimum energy in galactic coordinates. The 1 and 2-sigma total uncertainty bands are shown in green and yellow respectively. The expected value from isotropy considering the statistical (dotted line) and the statistical + systematic (solid line) uncertainties is also displayed. 


\section{References}

[1] M. Aguilar et al. [AMS Collaboration], Towards Understanding the Origin of Cosmic-Ray Positrons, Phys. Rev. Lett 122041102 (2019)

[2] M. Aguilar et al. [AMS Collaboration], Towards Understanding the Origin of Cosmic-Ray Electrons, Phys. Rev. Lett 122101101 (2019)

[3] D. Hooper, P. Blasi, and P. D. Serpico., Pulsars as the sources of high energy cosmic ray positrons, JCAP 01:25 (2009)

[4] T. Linden, and S. Profumo, Probing the Pulsar Origin of the Anomalous Positron Fraction with AMS-02 and Atmospheric Cherenkov Telescopes, APJ 77218 (2013)

[5] S. Manconi, M. Mauro, and F. Donato, Dipole anisotropy in cosmic electrons and positrons: inspection on local sources, JCAP 1701 (2017)

[6] Kun Fang, Xiao-Jun Bi, and Peng-Fei Yin, Discriminating local sources of high-energy cosmic electrons and positrons by current and future anisotropy measurements, Monthly Notices of the Royal Astronomical Society 478.4 (2018)

[7] M. Aguilar et al. [AMS Collaboration], First Result from the Alpha Magnetic Spectrometer on the International Space Station: Precision Measurement of the Positron Fraction in Primary Cosmic Rays of 0.5-350 GeV, Phys. Rev. Lett. 110141102 (2013)

[8] M. Aguilar et al. [AMS Collaboration], The Alpha Magnetic Spectrometer (AMS) on the international space station: Part II - Results from the first seven years, Physics Reports (2020)

[9] K. M. Gorski et al., HEALPix: A Framework for High-Resolution Discretization and Fast Analysis of Data Distributed on the Sphere, APJ 622759 (2005)

[10] M.A. Velasco, Ph.D Thesis, Universidad Complutense de Madrid, 2018; M. Molero, Ph.D Thesis, Universidad Autónoma de Madrid, 2021 